\title{
Predictive modelling of the impact of argon injection on H-mode plasmas in JET with the RITM code
}

\author{
B Unterberg ${ }^{1}$, D Kalupin ${ }^{1}$, M Z Tokar' ${ }^{1}$, G Corrigan ${ }^{2}$, P Dumortier ${ }^{3}$, \\ A Huber ${ }^{1}$, S Jachmich ${ }^{3}$, M Kempenaars ${ }^{4}$, A Kreter ${ }^{1}$, A M Messiaen ${ }^{3}$, \\ P Monier-Garbet $^{5}$, J Ongena ${ }^{3}$, M E Puiatti ${ }^{6}$, M Valisa ${ }^{6}$, \\ $M$ von Hellermann ${ }^{4}$ and Contributors to the EFDA-JET \\ Workprogramme ${ }^{7}$ \\ ${ }^{1}$ Institut für Plasmaphysik, Forschungszentrum Jülich GmbH, Ass. Euratom-FZ Jülich, D-52425 \\ Jülich, Germany ${ }^{8}$ \\ ${ }^{2}$ EURATOM UKAEA Fusion Association, Culham Science Centre, Abingdon, Oxon \\ OX14 3DB, UK \\ ${ }^{3}$ Laboratory for Plasma Physics, Ass. Euratom-Belgian State, Koninklijke Militaire \\ School-Ecole Royale Militaire, B-1000 Brussels, Belgium ${ }^{8}$ \\ ${ }^{4}$ FOM-Instituut voor Plasmafysica 'Rijnhuizen', Ass. Euratom-FOM, PO Box 1207, 3430 BE \\ Nieuwegein, The Netherlands ${ }^{8}$ \\ ${ }^{5}$ Ass. Euratom-CEA, DRFC, CEA Cadarache, St Paul lez Durance, France \\ ${ }^{6}$ Associazione Euratom-ENEA sulla Fusione, Consorzio RFX, Padova, Italy \\ E-mail: B.Unterberg@fz-juelich.de
}

Received 21 October 2003

Published 21 April 2004

Online at stacks.iop.org/PPCF/46/A241

DOI: $10.1088 / 0741-3335 / 46 / 5 \mathrm{~A} / 026$

\begin{abstract}
Self-consistent modelling of energy and particle transport of the plasma background and impurities has been performed with the code RITM for argon seeded high density H-mode plasmas in JET. The code can reproduce both the profiles in the plasma core and the structure of the edge pedestal. The impact of argon on core transport is found to be small; in particular, no significant change in confinement is observed in both experimental and modelling results. The same transport model, which has been used to reproduce density peaking in the radiative improved mode in TEXTOR, reveals a flat density profile in Ar seeded JET H-mode plasmas in agreement with the experimental observations. This behaviour is attributed to the rather flat profile of the safety factor in the bulk of H-mode discharges.
\end{abstract}

\section{Introduction}

Impurity radiation in the confined plasma and in the divertor is a possible tool to moderate the power flux to the plasma facing components. In addition, seeded impurities can provide

7 See appendix of paper by Pamela J et al 2002 19th IAEA Fusion Energy Conf. (Lyon, October 2002).

8 Partners in the Trilateral Euregio Cluster. 
a positive effect on energy confinement. In the radiative improved mode (RI-mode) at TEXTOR [1], a transition from L-mode to improved confinement (H89 up to 2) could be observed, which has been associated with the reduction of ion temperature gradient (ITG) driven transport [2,3]. A reduction of transport could also be observed in L-mode discharges with impurity seeding in JET [4,5] and DIII-D [6]. In H-mode discharges, high performance could be extended towards higher densities in JT-60U with argon injection [7]. In JET also argon injection has allowed to combine high density and increased radiation losses from the main chamber with good $\mathrm{H}$-mode performance [8]. To investigate the mechanisms at work, a transport code is necessary that treats heat and particle transport of the plasma background together with impurity transport and radiation in a self-consistent way. We present initial results of the application of the RITM code $[9,10]$ to $\mathrm{H}$-mode discharges at high densities with argon seeding in JET.

\section{RITM code}

The one-dimensional transport code RITM $[9,10]$ allows a self-consistent description of heat and particle transport in the entire cross-section of the plasma from the axis to the separatrix. The plasma shaping is taken into account by using the Shafranov shift calculated with inductively driven and bootstrap contributions to the current density and prescribed elongation and triangularity of magnetic surfaces. Continuity equations for electrons and impurity ions in all ionization stages are solved, including diffusive and convective components of the particle fluxes. The charged particle sources are determined by the ionization of neutrals generated by neutral beam injection (NBI) and those entering the confined volume across the separatrix. The behaviour of the latter in the plasma is described in a diffusive approximation. The flux and density of background ions is calculated under the assumption of quasi-neutrality. The external fuelling rates of background neutrals and of carbon and argon atoms are adapted to match the experimental volume averaged electron density and the total radiated power inside the confined plasma. The radiation profile is computed by taking all impurity ionization stages into account. This profile is used together with the experimental profiles of auxiliary heating from NBI and high frequency waves in the ion and electron heat transport equations to calculate the ion and electron temperature profiles. The boundary conditions at the separatrix are defined by the experimental decay lengths of the plasma parameters.

The transport coefficients include neoclassical pinch-velocities of the impurities and neoclassical ion heat diffusivity as well as anomalous contributions owing to drift wave instabilities. The latter are determined in mixing length approximation from the growth rate and perpendicular wave number of the most unstable modes. In the plasma core, transport driven by the toroidal ITG mode and dissipative trapped electron mode (DTE mode) is dominating while at the plasma edge transport owing to drift resistive ballooning and drift Alfvén (DA) modes is of importance. For the transport calculations shown in this paper, the transport coefficients have been used as described in [10] with the addition of transport owing to DA modes as given in [11]. In particular, the suppression of the DA instability is an important prerequisite to obtain an L-H transition. The suppression takes place if the normalized pressure increases and the collisionality decreases at the plasma edge with increased heating power as already discussed in [12]. The pressure gradient at the edge is limited by the ballooning instability threshold as given in [13]. The effect of the radial electric field on transport has not been taken into account in the present calculations.

To illustrate the capability of the code to reproduce the change of the edge profiles between $\mathrm{H}$ - and L-modes we show the variation of the total pressure profile (figure 1(a)) as well as the total ion heat diffusivity and the particle diffusivity from DA modes (figure $1(b)$ ) with the 


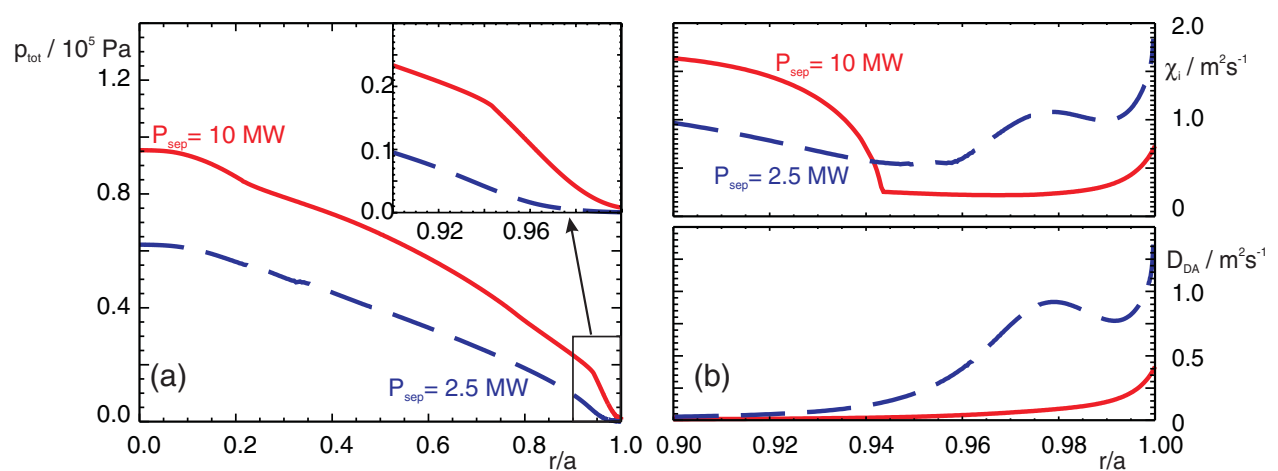

Figure 1. (a) Pressure profiles calculated for a power flux through the separatrix corresponding to H-mode (-) and L-mode stages (- - - ); inset shows pedestal region. (b) Total ion heat diffusivity and particle transport owing to DA modes in the pedestal region.

power flux across the separatrix. The global plasma parameters are taken from a strongly shaped high density H-mode discharge in the JET tokamak (JPN 53146, elongation $\kappa=1.7$, averaged triangularity $\delta=0.4$, plasma current $I_{\mathrm{P}}=2.3 \mathrm{MA}$, toroidal field $B_{\mathrm{T}}=2.4 \mathrm{~T}$ ) with a line averaged central electron density of $\bar{n}_{\mathrm{e}}=1.2 \times 10^{20} \mathrm{~m}^{-3}$ (cf [8] for details of the experiment). Taking the experimental heat source profiles corresponding to a power flux of $P_{\text {sep }}=10 \mathrm{MW}$ leads to an edge barrier with neoclassical ion heat transport between $r / a=0.94$ and $r / a=0.98$. For comparison, we reduced the power flux by a factor of 4 in the calculations which leads to an L-mode edge. A more refined description of the dynamics of the L-H transition, which probably will require the inclusion of $E \times B$ shear stabilization of the DA transport, is the subject of further investigations.

\section{Results of modelling for high density H-mode plasmas with argon injection}

A high triangularity H-mode scenario in JET with a normalized energy confinement time H98y 2 around 1 at the empirical Greenwald density limit was investigated. The plasma of the reference discharge without argon (JPN 53149) is auxiliary heated by $14 \mathrm{MW}$ of neutral beam and 2 MW of central ICRH. The injection of argon (JPN 53146) leads to an increased radiation fraction of $P_{\text {rad }} / P_{\text {heat }}=70 \%$ where $45 \%$ of the heating power is radiated from the edge of the main plasma [8].

\subsection{Profiles and transport characteristics of the background plasma}

In figure 2, the results of modelling and experimental data are compared: electron temperature $(a)$, density $(b)$ and pressure $(d)$ profiles are measured by the LIDAR Thomson scattering systems in the core and at the edge; the ion temperature profile $(c)$ corresponds to the fully stripped carbon ions measured by charge exchange recombination spectroscopy (CXRS). We note fair agreement between experiment and modelling in both the core and the pedestal regions. There is only a small difference between argon seeded and reference cases in both experimental and modelling data. Correspondingly, the transport coefficients owing to the different modes considered in the code are almost not affected by the argon ions. This is true for both the edge pedestal, where the radiation losses from argon ions do not modify the pressure considerably, and in the plasma core, where transport owing to ITG and DTE modes remains almost unchanged. 

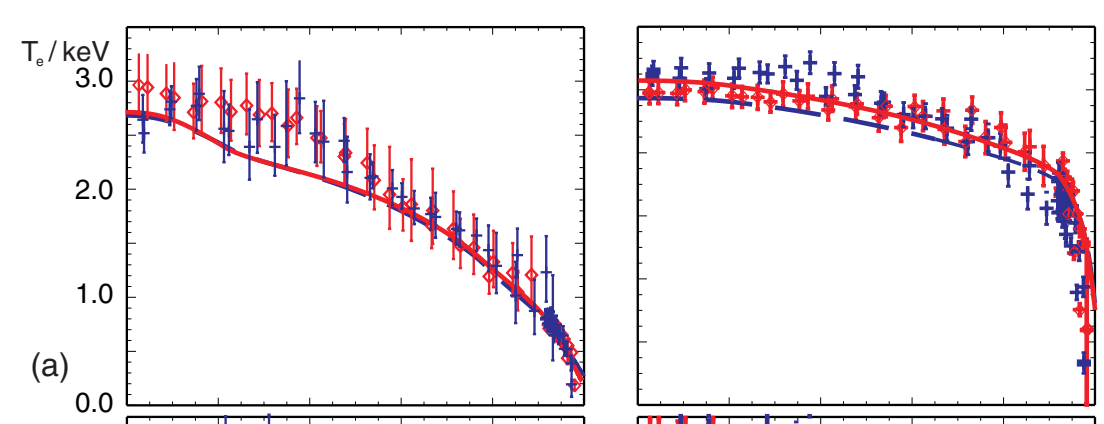

1.2

$n_{e} / 10^{20} m^{-3}$

0.8

0.4

(b)
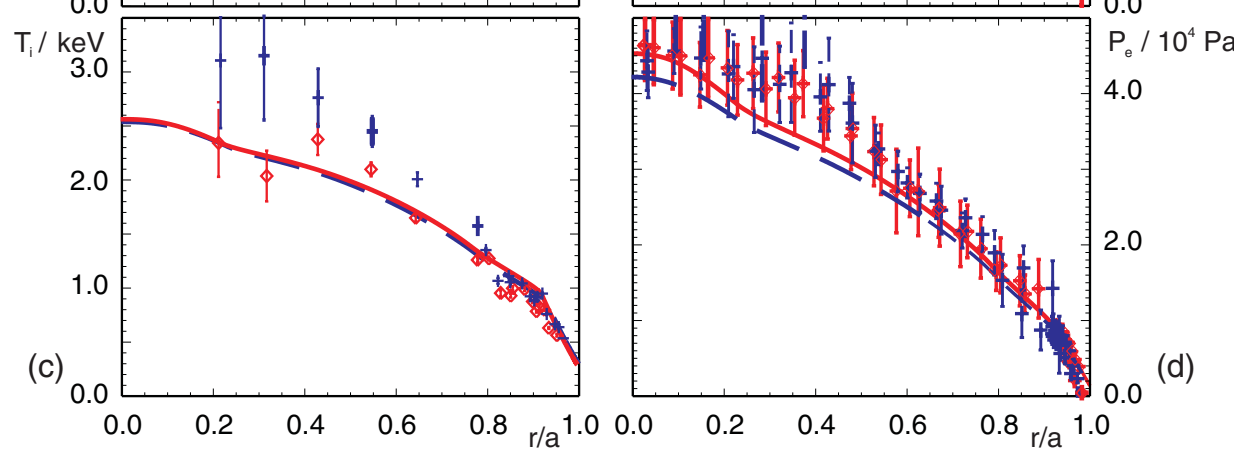

Figure 2. Experimental data and calculated profiles for argon seeded $\mathrm{H}$-mode $(\diamond /-)$ and unseeded reference (+/- - - -): (a) electron temperature, $(b)$ electron density, $(c)$ ion temperature, (d) electron pressure.
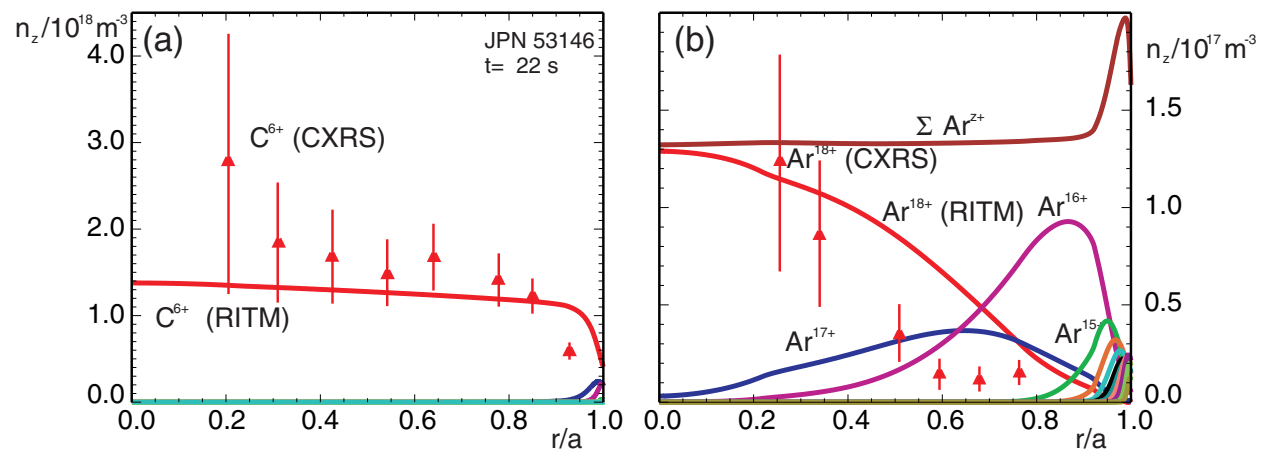

Figure 3. Radial profiles of different ionization stages of $(a)$ carbon and $(b)$ argon for H-mode plasma with argon seeding. Solid lines show results of modelling, triangles refer to experimental data from CXRS.

\subsection{Impurity transport and radiation}

The radial density distribution of the ionization stages of argon and carbon are depicted in figures $3(a)$ and $(b)$. Together with the results of our modelling, we show experimental data of the fully ionized nuclei $\mathrm{C}^{6+}$ and $\mathrm{Ar}^{18+}$ as measured by CXRS. The calculated profile of the carbon nuclei match the experimental profile fairly well within the error bars. However, the moderate steepening of $\mathrm{C}^{6+}$ ions as indicated from CXRS is not seen in the calculations. The contribution of lower ionization stages in the confined volume is negligible. Also, the calculated total density of all argon ions is flat throughout the entire profile indicating 


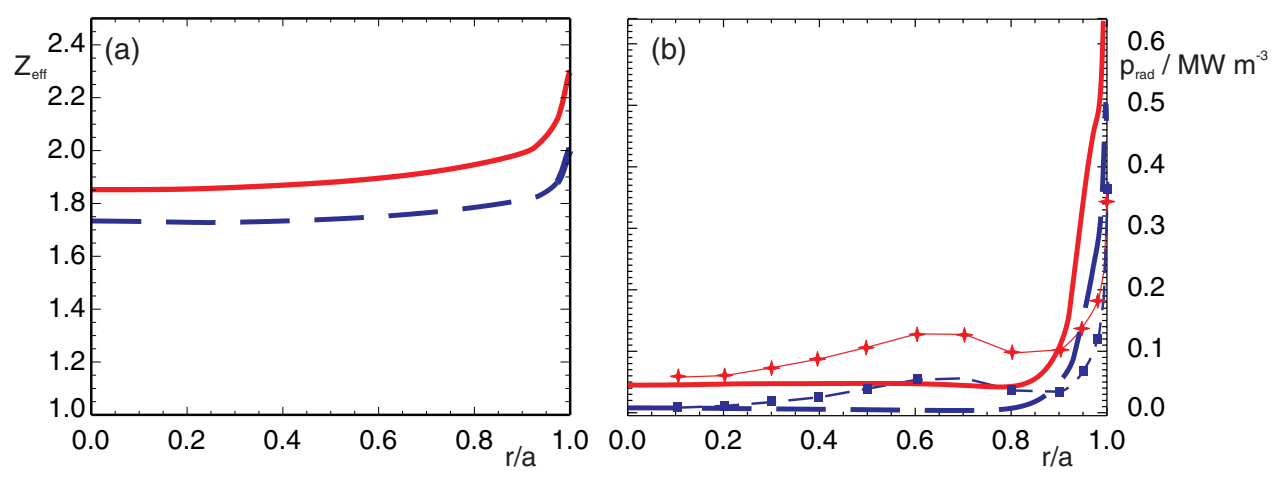

Figure 4. Radial profiles of (a) effective ion charge $Z_{\text {eff }}$ and $(b)$ radiated power density. Symbols refer to experimental data and lines show results of modelling with solid lines for the argon seeded $\mathrm{H}$-mode and dashed lines for the unseeded reference.

that the anomalous diffusion is dominating the neoclassical inward drift as could be expected because of the flat density profile of the background ions. The maximum at the edge can be related to a substantial neoclassical screening effect because of the steep temperature profile in the pedestal region as the argon ions are in the Pfirsch-Schlüter regime, where the temperature gradient driven fluxes are directed outward. While the argon concentration in the plasma centre, which is needed in the calculation to match the total radiation in the confined volume, is comparable to the experiment, the experimental profile of the argon nuclei is considerably steeper than the calculated one. This could be attributed to either an anomalous inward pinch or a too high diffusion coefficient for the impurity ions, which is the same as for the background in our modelling assumptions.

The $Z_{\text {eff }}$ profiles are shown in figure $4(a)$. The calculated profiles are in agreement with the line averaged values from the Bremsstrahlung measurements along a chord through the plasma centre, which give $\bar{Z}_{\text {eff }}=2.1$ for the argon seeded case and $\bar{Z}_{\text {eff }}=1.9$ for the unseeded reference. The distribution of the radiated power density is notably not poloidally symmetric in the experiment. Instead, there is a local maximum around the X-point. Therefore, the assumption of constant plasma parameters on flux surfaces within the one-dimensional calculations makes a comparison to the experiment difficult. We show in figure $4(b)$ the results of our calculations together with the flux surface average obtained from the two-dimensional tomographic reconstruction of bolometry. The values on axis are comparable in experimental and modelling data, but the experimental profile is considerably broader in the outer half of the plasma.

\section{Impact of $Z_{\text {eff }}$ on the density profile}

Finally, we investigate the impact of $Z_{\text {eff }}$ on particle transport and assess the question, whether a bifurcation to a peaked density profile and an associated improvement of energy confinement characteristic for the L-RI transition in TEXTOR [2] is feasible under conditions of H-mode in JET. In the transport model used in RITM, the density profile is governed by the ratio between the anomalous inward drift mainly governed by the DTE mode and the diffusion coefficient composed of contributions from DTE and ITG modes. The drift velocity $v_{\mathrm{DTE}}$ is related to the gradient length of the profile of the safety factor $L_{q}$ as $v_{\mathrm{DTE}}=-D_{\mathrm{DTE}} / L_{q}$.

From the local heat and particle balance the gradient lengths of density and temperature, $L_{n}$ and $L_{\mathrm{T}}$ can be deduced. At the high density under consideration, we assume $T_{\mathrm{e}}=T_{\mathrm{i}}$ 


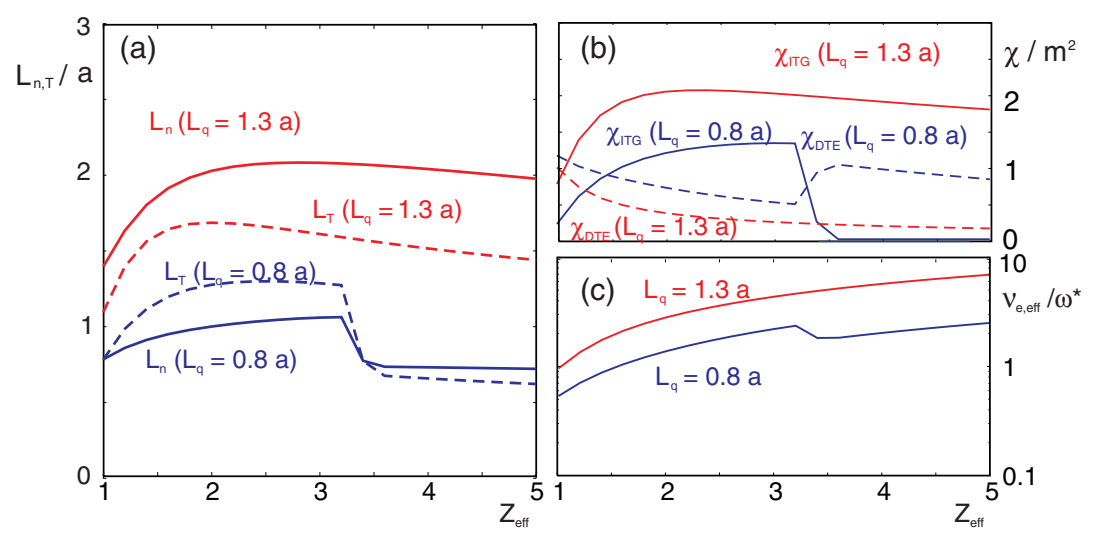

Figure 5. Variation of transport characteristics with $Z_{\mathrm{eff}}$ for two different gradients of the safety factor (experimental $L_{q}=1.3 a$ and hypothetical $L_{q}=0.8 a$ ): (a) decay lengths of temperature (- - - ) and density (- $\left.{ }_{-}\right)$, (b) heat diffusivity related to ITG (-) and DTE modes (- - - ), (c) normalized collision frequency.

and $L_{n_{\mathrm{e}}}=L_{n_{\mathrm{i}}}$. Figure 5(a) shows the $Z_{\mathrm{eff}}$ dependence of $L_{n}$ and $L_{\mathrm{T}}$ computed at the radial position $r / a=2 / 3$ with $L_{q}=1.3 a=1.3 m$ inferred from the experimental data ( $n=9 \times 10^{19} \mathrm{~m}^{-3}, T=1820 \mathrm{eV}$, heat flux density $q=0.061 \mathrm{MW} \mathrm{m}^{-2}$ and particle flux density $\Gamma=10^{19} \mathrm{~m}^{-2} \mathrm{~s}^{-1}$ ). We note that with increasing $Z_{\text {eff }}$ the density profile rather slightly flattens than steepens as could be expected because $v_{\text {DTE }}$ reduces with increasing collision frequency of trapped electrons normalized to the drift frequency, $v_{\mathrm{e}, \text { eff }} / \omega^{*}$ (cf figure $5(c)$ ). With respect to energy transport, the ITG mode is dominating, but the corresponding $\chi_{\mathrm{i} \text {, ITG }}$ only varies weakly in the $Z_{\text {eff }}$ range under consideration (figure $5(b)$ ). This fact explains the negligible variation of the profile shapes as discussed in section 3.1. Even at much higher $Z_{\text {eff }}$ no bifurcation occurs. This situation changes if we redo the calculations with a smaller gradient length of the $q$-profile $\left(L_{q}=0.8 a\right)$ being more typical for L-mode discharges. Here, we find a bifurcation to a state with peaked density profiles where the ITG mode is suppressed at $Z_{\text {eff }} \approx 3.2$. The absolute value of $Z_{\text {eff }}$ for the transition is dependent on the particle flux density determined by the integrated particle source inside the radial position under consideration.

Note that in JET a transition to a state with steeper density profiles has been observed and successfully modelled with RITM in low density, diverted L-mode plasmas with substantial neon seeding [5]. These discharges being L-mode have a substantially smaller $L_{q}$ than the $\mathrm{H}$-mode discharges considered in this paper.

\section{Conclusions and outlook}

Predictive and self-consistent modelling of the impact of argon on high density H-mode plasmas has been performed. Fair agreement between modelling results and experimental data was obtained. It was found that in this plasma regime the injected impurities only weakly affect the background transport. In particular, a transition to a state with peaked density profiles and improved core confinement as observed in L-mode plasmas is hindered by the very flat $q$-profile in these $\mathrm{H}$-mode plasmas. Modelling of impurity transport remains a challenge as the experimental data indicate a stronger inward transport as the modelling shows. Extensive comparison to experimental data is needed to further test the assumptions made in the model. 


\section{References}

[1] Messiaen A M et al 1996 Phys. Rev. Lett. 772487

[2] Tokar' M Z et al 2000 Phys. Rev. Lett. 84895

[3] Tokar' M Z et al 1999 Plasma Phys. Control. Fusion 41 L9

[4] Jackson G L et al 2002 Plasma Phys. Control. Fusion 441893

[5] Tokar' M Z et al 2002 Plasma Phys. Control. Fusion 441903

[6] Jackson G L et al 2002 Nucl. Fusion 4228

[7] Kubo H et al 2001 Nucl. Fusion 41227

[8] Dumortier P et al 2002 Plasma Phys. Control. Fusion 441845

[9] Tokar' M Z 1994 Plasma Phys. Control. Fusion 361819

[10] Kalupin D et al 2001 Plasma Phys. Control. Fusion 43945

[11] Kerner W et al 1998 Contrib. Plasma Phys. 38118

[12] Janeschitz G et al 1999 J. Nucl. Mater. 266-299 843

[13] Onjun T et al 2002 Phys. Plasmas 95018 